\title{
VIABILIDADE ECONÔMICA DA RECICLAGEM DOS RESÍDUOS DOMÉSTICOS DA CIDADE DE COIMBRA - UTILIZANDO O APLICATIVO VERDES-PT
}

\section{ECONOMIC FEASIBILITY OF RECYCLING DOMESTIC WASTE FROM THE CITY OF COIMBRA - USING THE GREEN-EN APPLICATION}

\section{Marcio Magera Conceição ${ }^{1}$, Joelma Telesi Pacheco Conceição², Alessandro Rezende da Silva ${ }^{3}$, Maria Fatima} Alves ${ }^{4}$, Marcelo Dourado Sales ${ }^{5}$, Maurício Lamano Ferreira ${ }^{6}$

\section{Submetido: $16 / 02 / 2021$}

Aprovado: 28/02/2021

\section{RESUMO}

Este trabalho irá mostrar o funcionamento e aplicabilidade do software VERDES-PT, usando a cidade de Coimbra como exemplo. A geração de resíduos sólidos domésticos vem aumentando muito nas últimas décadas, os órgãos públicos responsáveis pela gestão destes resíduos, vem adotando a reciclagem como uma das formas de reutilizar o lixo urbano, não somente como matéria prima, mas também como gerador de emprego e renda a classes com menos formação educacional. A iniciativa privada, juntamente com governos locais, estão juntos em projetos de implantação de usinas de reciclagem. A cidade de Coimbra tem um potencial anual de $€ 31.017 .431,00$ milhões de euros, quando se aplica a logística reversa, utilizando o VERDES -PT, estes valores poderiam voltar aos cofres públicos, se fosse adotado a coleta seletiva para $100 \%$ da sua geração de lixo urbano e ele fosse reciclado. Mas, como será visto neste artigo, a cidade perde 14 milhões de euros por ano por não adotar uma política de gestão de resíduos que aproveite o total da sua geração, mas mesmo assim, ainda gera 2.362 empregos de um salário mínimo por ano com a reciclagem dos resíduos urbanos já implantada na cidade.

PALAVRA-CHAVE: Reciclagem. Coleta seletiva. Emprego. Economia. Logística reversa

\begin{abstract}
This work will show the operation and applicability of the VERDES-PT software, using the city of Coimbra as an analysis. The generation of solid domestic waste has been increasing a lot in the last decades, the public agencies responsible for the management of this waste, have been adopting recycling as one of the ways to reuse urban waste, not only as raw material, but also as a generator of jobs and income to classes with less educational background. The private sector, together with local governments, are together in projects to implement recycling plants. The city of Coimbra has an annual potential of $€ 31,017,431.00$ million euros, when reverse logistics is applied, using VERDES $P T$, these values could be returned to public coffers, if the selective collection for $100 \%$ of the its
\end{abstract}

\footnotetext{
1 Economista pela PUC- Campinas. MBA de Marketing - ESAMC, Sorocaba. Mestrado em Administração pela UNG - Guarulhos. Mestrado em Sociologia pela PUC - São Paulo. Doutorado em Sociologia pela PUC - São Paulo. Doutorado em Administração pela FCU - USA. Pós Doutor Unicamp - Campinas. Pós Doutor FCU - USA. Pós Doutor UC- Portugal. Jornalista e Escritor. Avaliador do MEC/INEP. Pró-Reitor da Universidade de Guarulhos, SP. Editor-chefe da RECIMA21 - REVISTA CIENTÍFICA MULTIDISCIPLINAR. https://orcid.org/0000-0001-6477-4580 -rrecima21@gmail.com

2 Professor Universitária há mais de 10 anos. Mestrado em Administração de Empresas pela FACCAMP. https://orcid.org/0000-0002-7045-6260 - joelma.telesi@gmail.com

3 Professor universitário mestre e doutor em Ciências Sociais - https://orcid.org/0000-0002-5381-2155 alessandroligadf@gmail.com

4 Professora e vice coordenadora do CFE da Universidade de Coimbra - https://orcid.org/0000-0003-2600-8652 fatimaa@uab.pt

5 Professor universitário mestre e doutor em administração de empresas pela FCU, USA e FACCAMP. https://orcid.org/0000-0003-3507-1143 - madourado2309@gmail.com

6 Professor universitário mestre e doutor - UNG Universidade Guarulhos - https://orcid.org/0000-0002-7647-3635 - mauecologia@yahoo.com.br
} 


\section{RECIMA21 - REVISTA CIENTÍFICA MULTIDISCIPLINAR}

VIABILIDADE ECONÔMICA DA RECICLAGEM DOS RESÍDUOS DOMÉSTICOS DA CIDADE DE COIMBRA - UTILIZANDO O APLICATIVO VERDES-PT Marcio Magera Conceição, Joelma Telesi Pacheco Conceição, Alessandro Rezende da Silva, Maria Fatima Alves, Marcelo Dourado Sales, Maurício Lamano Ferreira

generation of urban waste. But, as will be seen in this article, the city loses 14 million euros per year, for not adopting a waste management policy that takes full advantage of its generation, but even so, it still generates 2,362 minimum wage jobs per year with the recycling of urban waste already implemented in the city.

KEYWORDS: Recycling. Selective collect. Job. Economy. Reverse logistics

\section{INTRODUÇÃO}

Nos últimos 70 anos, a geração dos resíduos sólidos domésticos vem aumentando em todo o mundo. Isto é, sem dúvidas, o resultado do aumento do consumo de materiais descartáveis e obsoletos, a vida útil das embalagens e dos produtos não completam o ciclo da garantia deles, e esta é a lógica do capitalismo do século XX. Ecologistas, governos e instituições alardeiam aos quatros cantos que chegaremos, num futuro próximo, a uma situação de calamidade pública, tanto pela geração dos resíduos, cujas consequências já são sentidas nos aterros e na falta deles, quanto pela escassez de recursos naturais para a geração destes produtos, que, segundo a ONU, já se encontra com um déficit de $40 \%$ e vem aumentando $2,5 \%$ ao ano (MAGERA, 2013). Uma das alternativas que estão sendo propostas para minimizar esse quadro é a gestão da reciclagem de lixo urbano, cujos maiores incentivadores estão sendo os governos municipais e algumas Ongs. Os avanços nos índices de reciclagem estão ligados muito mais à questão mercadológica, como é o caso da latinha de alumínio, sem deixar de salientar o desemprego e exclusão social, do que a uma conscientização ambiental universal por parte dos agentes envolvidos neste segmento. A pressão internacional levada pelos países desenvolvidos tem ajudado neste sentido, mas a falta de políticas públicas direcionadas para a criação de usinas de reciclagem dos resíduos urbanos não está trazendo resultados promissores neste segmento. Enquanto a matéria prima for mais barata do que os produtos reciclados, esta lógica não se reverterá tão cedo. A logística reversa, se considerada apenas o produto triado e não sua economia de todo o processo, não traz lucro para o capital investido. Mas este artigo, busca mostrar que, se considerada toda a cadeia produtiva, a reciclagem dos resíduos urbanos traz resultados positivos não só para o meio ambiente, mas para todos os envolvidos no processo.

A cidade de Coimbra vem adotando a reciclagem através da coleta seletiva dos resíduos urbanos desde o início da década de 80, principalmente após a entrada de Portugal na Comunidade Europeia. Mas, somente uma pequena parte vai para as usinas de reciclagem, a grande maioria dos resíduos urbanos gerados, $60 \%$ ainda vai para os aterros sanitários ou são queimados para a geração de energia, em pontos espalhados por todo o país. Este trabalho visa levantar os números econômicos da reciclagem formal da cidade de Coimbra e seus impactos macroambientais, utilizando o aplicativo VERDES PT para mensurar seus dados. 


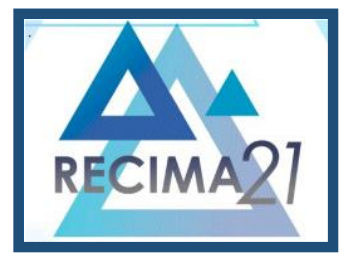

\section{RECIMA21 - REVISTA CIENTÍFICA MULTIDISCIPLINAR}

VIABILIDADE ECONÔMICA DA RECICLAGEM DOS RESÍDUOS DOMÉSTICOS DA CIDADE DE COIMBRA - UTILIZANDO O APLICATIVO VERDES-PT Marcio Magera Conceição, Joelma Telesi Pacheco Conceição, Alessandro Rezende da Silva, Maria Fatima Alves, Marcelo Dourado Sales, Maurício Lamano Ferreira

Coimbra é uma cidade da parte central de Portugal, no ano 2020 contava com uma população de 135 mil habitantes, segundo o Pordata (2020), lá está concentrado também uma boa parte do PIB do país, mais de $20 \%$ na região (INE, 2020). Coimbra é uma cidade universitária, desde a fundação da Universidade de Coimbra em 1 de março do ano de 1290 . Atualmente, mais de $20 \%$ da população da cidade tem ligação com a universidade, direta ou indiretamente.

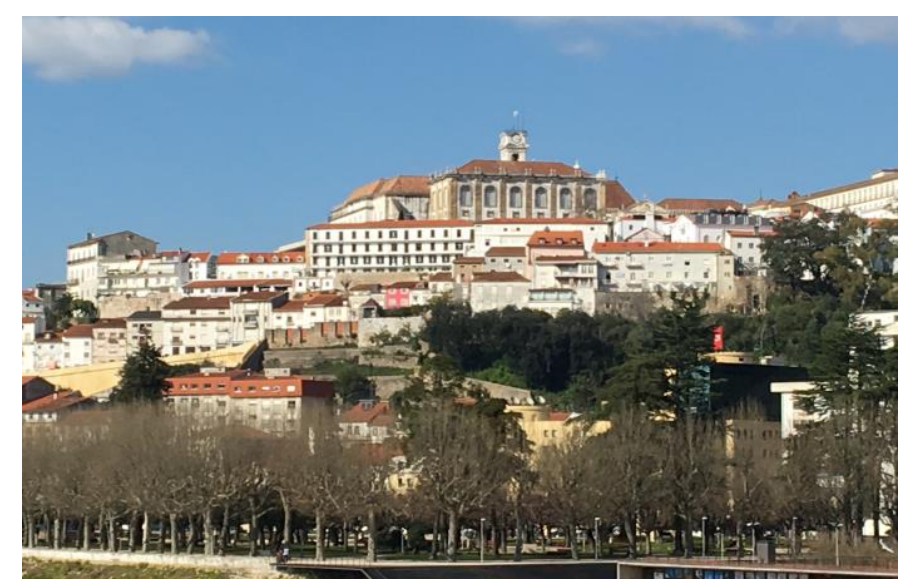

Foto: cidade de Coimbra, autor, 2018.

A produção anual de resíduos sólidos domésticos de Portugal é de 4.500 .000 toneladas, sendo $86 \%$ recolha indiferenciada e $14 \%$ recolha seletiva. A geração per capita do país é de 452 kg.hab.ano ou 1,24 kg.hab.dia, mas a média da cidade de Coimbra é de $486 \mathrm{~kg}$.hab.ano ou 1,35 kg.hab.dia, o que supera a média nacional. O que potencializa a geração de lixo urbano na cidade é a concentração do PIB nas regiões metropolitanas de países desenvolvidos, fato muito comum em países semelhantes da Comunidade Europeia (INE, 2020), e também o fato da cidade viver em torno da Universidade de Coimbra, que recebe alunos de todo o país e também da Europa, com alta concentração de renda, o que proporciona mais consumo e descarte (MAGERA, 2018).

O lixo doméstico urbano é um grande problema para muitas capitais do mundo, mas se houvesse uma política de reciclagem de resíduos urbanos domésticos, teríamos uma economia de recursos naturais, bem como uma geração de emprego e renda a milhões de desempregados em muitos países, sem contar com a diminuição da contaminação destes resíduos que são alocados em lugares inapropriados e sem fiscalização, contaminando o solo e o ar. Segundo estudos de Magera (2020), a cada tonelada reciclada teríamos uma economia de U\$100,00 dólares para os cofres públicos, e a geração de um emprego de $\bigcup \$ 200,00$ dólares por mês. Este projeto ajudaria a colocar no mercado de trabalho as pessoas com baixa escolarização, visto que o trabalho nas usinas de reciclagem, não exige uma formação técnica para sua atuação. Estes dados refletem a média do mercado de reciclagem do Brasil, enquanto que, para Portugal, esta média corresponde a U\$300,00 


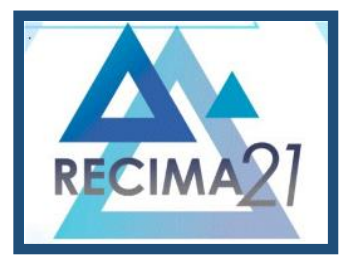

\section{RECIMA21 - REVISTA CIENTÍFICA MULTIDISCIPLINAR}

VIABILIDADE ECONÔMICA DA RECICLAGEM DOS RESÍDUOS DOMÉSTICOS DA CIDADE DE COIMBRA - UTILIZANDO O APLICATIVO VERDES-PT Marcio Magera Conceição, Joelma Telesi Pacheco Conceição, Alessandro Rezende da Silva, Maria Fatima Alves, Marcelo Dourado Sales, Maurício Lamano Ferreira

o valor da tonelada, considerando o mix médio dos produtos reciclados e comercializados no mercado.

O objetivo deste artigo é mostrar quanto de valor econômico a cidade de Coimbra poderia obter com geração de emprego e renda se realizasse uma política de gestão de reciclagem de resíduos urbanos domésticos mais abrangente na cidade. Para obter estes dados/números utilizouse o aplicativo VERDES -PT - Viabilidade Econômica dos Resíduos Sólidos de Portugal, cujo registro é ISNB 978-85-903805-3-5. Este aplicativo é o resultado de um pós-doutoramento realizado pelo professor Márcio Magera Conceição na Universidade de Coimbra no ano de 2018. O estudo foi desenvolvido no Centro de Ecologia Funcional CEF, com a orientação da Dra. Maria de Fátima Alves, vice-coordenadora do departamento de pesquisa ecológica.

\section{METODOLOGIA DO VERDES - PT}

As cidades infelizmente, desconhecem a geração e a composição dos resíduos sólidos urbanos domésticos, ficando o agente público e privado sem saber como calcular a potencialidade da geração dos materiais que ele pretende reciclar e o quanto investir em infraestrutura. Portanto, surge a importância de existir um aplicativo baseado em dados científicos, possibilitando o acesso fácil para o desenvolvimento de informações precisas, e salientando que o aplicativo VERDES - PT é gratuito, dando acesso a todos que necessitam destas informações para gerenciar seus negócios focados neste segmento de resíduos sólidos domésticos urbano.

O aplicativo VERDES -PT, apresenta aos agentes deste segmento, uma facilidade em realizar várias situações e composições de resultados da reciclagem dos cinco principais produtos que compõem os resíduos urbanos da sociedade nos dias atuais, tais como: lata de alumínio, lata de aço, papel e papelão, plástico e vidro. Esses produtos somam mais de $90 \%$ dos valores mercadológicos dos produtos reciclados da cidade de Coimbra; sendo assim, o aplicativo procura dar aos empreendedores e governos um resultado de viabilidade econômica desse empreendimento ambiental justo socialmente e sustentável, tanto em uma visão macroambiental quanto mercadológica. $\mathrm{O}$ aplicativo é fácil de usar e contém toda a metodologia, onde o usuário poderá criar várias situações com resultados científicos importantes para a tomada de decisões, tanto no setor público, quando no privado para o investimento nas usinas de reciclagem dos resíduos domésticos urbanos. 


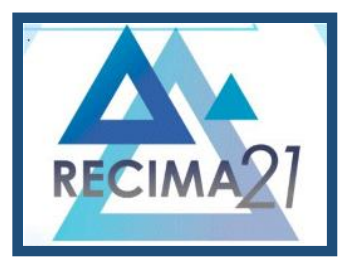

\section{RECIMA21 - REVISTA CIENTÍFICA MULTIDISCIPLINAR}

\section{VIABILIDADE ECONÔMICA DA RECICLAGEM DOS RESÍDUOS DOMÉSTICOS DA CIDADE DE COIMBRA} - UTILIZANDO O APLICATIVO VERDES-PT Marcio Magera Conceição, Joelma Telesi Pacheco Conceição, Alessandro Rezende da Silva, Maria Fatima Alves, Marcelo Dourado Sales, Maurício Lamano Ferreira
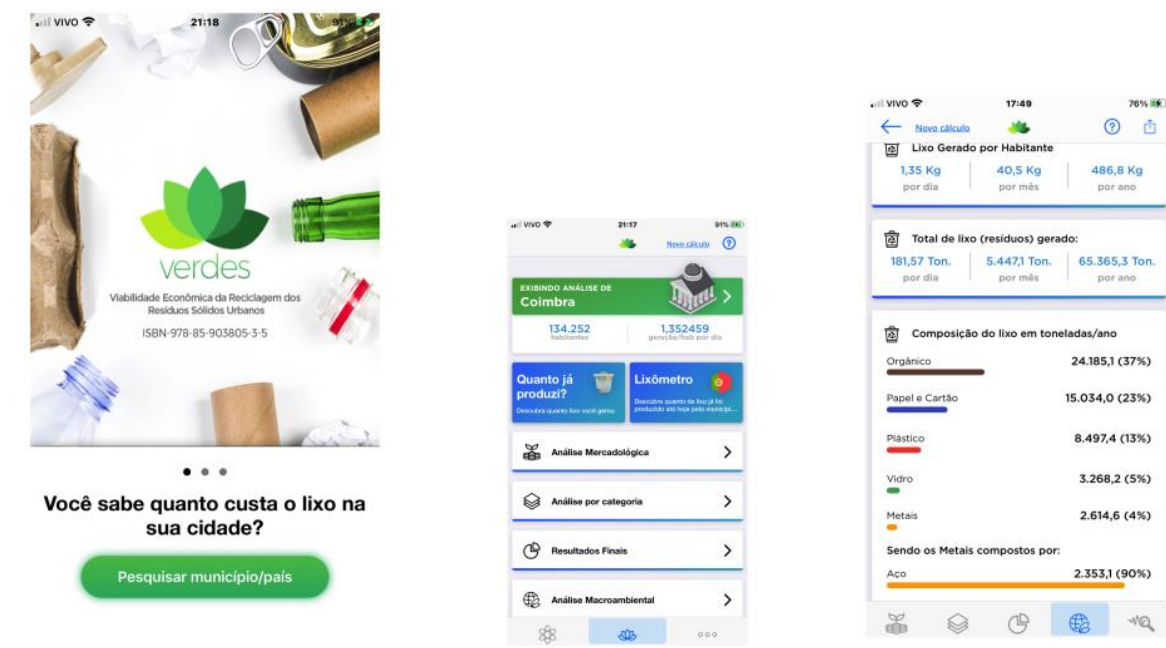

Prints da tela do Aplicativo VERDES-PT.

O aplicativo dará ao usuário todas as informações que se referem à viabilidade da reciclagem dos resíduos sólidos urbanos, e ele poderá ser impresso para futuras utilizações, ou ter salvo seus dados em análise, no diretório indicado.

O aplicativo VERDES- PT disponibilizará os seguintes dados de viabilidade econômica macroambiental:

c Geração de lixo per capita dia, por mês e ano.

c Geração total de lixo dia, por mês e ano.

c Economia anual e mensal possível em matéria-prima, energia, água.

c Economia anual e mensal perdida pela não-reciclagem.

c Economia anual e mensal obtida pela reciclagem formal/informal.

c Geração de empregos de um salário mínimo mensal possível.

c Geração de empregos de um salário mínimo mensal obtido.

c Empregos de um salário mínimo mensal perdidos.

c Dados econômicos e físicos de cada produto, isoladamente: lata de alumínio, papel e papelão, plástico, vidro e lata de aço.

c Resultado total da viabilidade econômica obtida e perdida com a aplicação do processo de reciclagem.

Na análise mercadológica o programa disponibilizará:

c Resultados, em toneladas, gerados dos cinco produtos, parciais e totais. 


\section{RECIMA21 - REVISTA CIENTÍFICA MULTIDISCIPLINAR}

VIABILIDADE ECONÔMICA DA RECICLAGEM DOS RESÍDUOS DOMÉSTICOS DA CIDADE DE COIMBRA - UTILIZANDO O APLICATIVO VERDES-PT Marcio Magera Conceição, Joelma Telesi Pacheco Conceição, Alessandro Rezende da Silva, Maria Fatima Alves, Marcelo Dourado Sales, Maurício Lamano Ferreira

C Valor mercadológico dos cinco produtos reciclados.

c Preço médio por tonelada dos produtos.

c Resultado econômico potencial a ser conseguido com o processo.

c Geração de emprego de um salário mínimo por mês.

A metodologia utilizada neste aplicativo tem como princípio a matemática mercadológica dos recursos naturais utilizados na composição dos produtos em análise (lata de alumínio, lata de aço, plástico, papel e papelão e vidro), bem como os preços mercadológicos dos serviços para a transformação até o consumidor final, utilizando-se, neste contexto, os preços dos serviços públicos e privados que se referem à coleta e ao processo de reciclagem dos resíduos sólidos, sem deixar de se considerar os custos do processo produtivo. Uma parte do programa está baseada no modelo proposto por Thomas Duston, no livro How to measure the gains from recycling.

Os resultados são obtidos analisando os mais lucrativos produtos reciclados do lixo urbano de Portugal, que são: lata de alumínio, papel e papelão, plástico, vidro e lata de aço, os quais representam mais de $90 \%$ dos valores mercadológicos. Com base na quantia produzida, reciclada e disposta em aterros, torna-se possível mensurar a economia de matéria-prima, energia, água e a redução dos danos ambientais, coleta, transporte e arranjo final do lixo. Com a quantia de lixo jogada nos aterros, somada aos índices que foram reciclados temos o total produzido ou consumido de um determinado produto.

Tanto os valores em Mwh - Megawatts hora - da energia elétrica, bem como em metro cúbico da água e a matéria-prima utilizada na composição dos produtos reciclados, foram levantados no mercado fornecedor de Portugal. $\mathrm{Na}$ análise macroambiental os valores dos produtos reciclados vendidos não foram utilizados para efeito de cálculo, visto que quando se considera a compra desses produtos os valores se anulam. Para efeito de cálculo foi utilizado o custo do processo de reciclagem, que é definido a partir do custo de transporte, armazenamento, enfardamento, trituração, lavagem, custos administrativos, que entram na fórmula com sinal negativo. Os custos evitados com a coleta, transporte e disposição final do lixo entram como sinal positivo, visto representar uma economia com o processo de reciclagem adotado.

O ganho econômico com a energia elétrica, matéria-prima e água é computado individualmente em cada planilha de cálculo dos materiais em estudo, depois elas são somadas e temos o resultado final, subtraindo-se o custo do processo de reciclagem.

Os principais indicadores utilizados nesta fórmula para se achar a viabilidade econômica da reciclagem dos resíduos sólidos da cidade de Coimbra foram:

c População do local em estudo

c. Produção do lixo urbano 


\section{RECIMA21 - REVISTA CIENTÍFICA MULTIDISCIPLINAR}

VIABILIDADE ECONÔMICA DA RECICLAGEM DOS RESÍDUOS DOMÉSTICOS DA CIDADE DE COIMBRA - UTILIZANDO O APLICATIVO VERDES-PT Marcio Magera Conceição, Joelma Telesi Pacheco Conceição, Alessandro Rezende da Silva, Maria Fatima Alves, Marcelo Dourado Sales, Maurício Lamano Ferreira

C Composição do lixo urbano em porcentagem

c Tarifa de energia elétrica média cobrada para as classes sociais

(c) Peso da lata de alumínio

c Economia de energia elétrica obtida na produção por meio da reciclagem da lata de alumínio

e Índice de reciclagem da lata de alumínio

c Consumo per capita de latas de alumínio em Portugal

c Custo da tonelada de bauxita

c Composição de uma tonelada de alumínio

e Redução na poluição da água em $97 \%$

c Redução na poluição do ar em $95 \%$

c Índice de reciclagem de vidro

c Quantidade anual estimada de vidro para embalagem

c Quantidade anual estimada da reciclagem do vidro

C Produção a partir da reciclagem

c Consumo de energia

c Economia de energia a partir da produção com cacos

c Custo da fabricação do vidro Soda-Cal a partir da mistura com os cacos

c Redução na poluição do ar em $20 \%$

(c) Redução no consumo de água em $50 \%$

C Índice de reciclagem de papel e papelão

c Consumo de energia elétrica na produção da tonelada de papel a partir da matéria-prima

e Economia de água para cada tonelada de papel reciclada

(c) Redução na poluição da água em $35 \%$

c Redução na poluição do ar em $74 \%$

c Índice de reciclagem do plástico

e Consumo de energia elétrica na produção da tonelada do plástico

c Consumo de energia elétrica a partir de material reciclado

e Preço da tonelada de plástico em Lisboa, usando-se como base as resinas termoplásticas que compõem o produto

C Redução da poluição do ar em $80 \%$

c Redução na poluição da água em $40 \%$

c. Índice da reciclagem da lata de aço em Portugal

c Quantidade de lata de aço consumida per capita

e Preço da tonelada de ferro-gusa

C Redução no consumo da água com a reciclagem

c. Valor do metro cúbico da água 


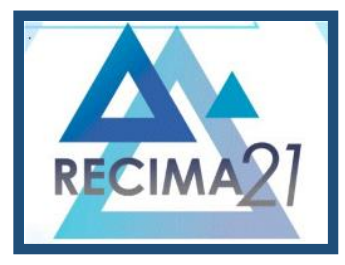

\section{RECIMA21 - REVISTA CIENTÍFICA MULTIDISCIPLINAR}

VIABILIDADE ECONÔMICA DA RECICLAGEM DOS RESÍDUOS DOMÉSTICOS DA CIDADE DE COIMBRA - UTILIZANDO O APLICATIVO VERDES-PT Marcio Magera Conceição, Joelma Telesi Pacheco Conceição, Alessandro Rezende da Silva, Maria Fatima Alves, Marcelo Dourado Sales, Maurício Lamano Ferreira

c Economia de energia elétrica a partir da reciclagem

c Redução no consumo de água com a reciclagem em $40 \%$

c Redução na poluição do ar com a reciclagem em $85 \%$

e Redução na poluição da água com a reciclagem em $76 \%$

\section{RESULTADOS FINAIS}

O resultado total de euros obtidos pela aplicação da reciclagem formal dos resíduos sólidos urbanos da cidade de Coimbra é de $€ 16.436 .805,49$. Já o total perdido pela não reciclagem, material que foi jogado nos aterros sanitários ou queimado para gerar energia elétrica é de $€ 14.580 .626,29$. Usando como referência o valor que foi reciclado, a geração de emprego foi de 2.362 mil e a perda de geração de emprego pela não reciclagem foi de 2.059 mil. Estes valores saíram do custo da reciclagem que foi: $€ 3.344 .240,01$, sendo o custo evitado com a coleta: $€ 953.727,71$, e o ganho de energia elétrica: $€ 3.714 .508,52$. Ganho de matéria-prima: $€ 14.484 .065,50$. Ganho de água: $€$ $628.743,76$.

Os impactos macroambientais positivos causados pela reciclagem, podem ser medidos com a economia dos produtos naturais que deixaram de ser extraídos. Em um ano de reciclagem a cidade de Coimbra poupou 210.476 mil árvores, que deixaram de ser cortadas pelo processo de reutilização das matérias primas, que antes iriam para os aterros e que neste processo de reciclagem, acabam tendo uma segunda vida no mercado.

A reciclagem economizou no processo 167,3 toneladas de bauxita, matéria prima das latinhas de alumínio. Neste processo economiza-se também energia elétrica, que foi de $€ 155.538,08$, bem como milhões de metros cúbicos de água. Mesmo assim, os cidadãos de Coimbra jogaram mais de 6.033.722 milhões de latinhas de alumínio nos aterros sanitários, um desperdício de milhões de euros que poderiam ir para os cofres públicos.

A geração por tipo de resíduos, segundo o aplicativo VERDES -PT foi de:

Orgânica: 24.185,1 (37\%)

Papel e Cartão: 15.034,0 (23\%)

Plástico: 8.497,4 (13\%)

Vidro: $3.268,2(5 \%)$

Metais: $2.614,6(4 \%)$

O destino dos resíduos sólidos urbanos da cidade de Coimbra, segundo o aplicativo VERDES-PT é:

Aterro: $18.955,9(29 \%)$

Tratamento MB: 18.302,2 (28\%) 


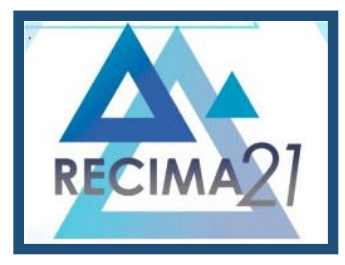

\section{RECIMA21 - REVISTA CIENTÍFICA MULTIDISCIPLINAR}

VIABILIDADE ECONÔMICA DA RECICLAGEM DOS RESÍDUOS DOMÉSTICOS DA CIDADE DE COIMBRA - UTILIZANDO O APLICATIVO VERDES-PT Marcio Magera Conceição, Joelma Telesi Pacheco Conceição, Alessandro Rezende da Silva, Maria Fatima Alves, Marcelo Dourado Sales, Maurício Lamano Ferreira

Valorização do Material: 7.190,1 (11\%)

Valorização Energética: 14.380,3 (22\%)

Tratamento Mecânico: 5.229,2 (8\%)

Valorização Orgânica: 1.307,3 (2\%)

Analisando os dados do VERDES -PT para a cidade de Coimbra, nota-se que é viável economicamente a reciclagem dos resíduos sólidos urbanos domésticos. Estamos falando de uma economia de 16,5 milhões de euros por ano, e uma inclusão social de mais de 2,5 mil novos empregos neste segmento, sem levar em consideração a economia de recursos naturais e a não contaminação dos rios, solos e ar com o destino destes resíduos de forma inadequada, como vem sendo realizado por políticas públicas, que não levam os interesses do Meio Ambiente como algo saudável para a população.

O fato de Portugal ser um grande importador de matéria prima e produtos acabados, mostra a importância de se utilizar da reciclagem para poupar e melhorar o resultado da sua balança comercial, que é quase sempre deficitária, segundo a Tradingeconomics 2021.

O aplicativo mostra também a perda anual de 14,5 milhões de euros que são jogados nos aterros em forma de embalagens ou matérias primas que poderiam ser reutilizadas, mas acabam sem nenhum aproveitamento e o pior, a população ainda paga para coletar e depositar este material nos aterros sanitários do país. Este fato perverso pode mudar, se as autoridades públicas do país adotassem de maneira mais séria a reciclagem como uma alternativa econômica e ambiental para resolver parte dos seus problemas sociais que se agravaram muito com a chegada da Pandemia do Covid-19 no ano de 2020. Espera-se que Portugal siga seus vizinhos, que segundo a Comissão Europeia para tratamento dos resíduos urbanos, vem conseguindo lucros de mais de 100 bilhões de dólares por ano com a reciclagem de resíduos. https://www.dw.com/pt-br/tratamento-de-lixo-naalemanha-está-entre-os-mais-eficientes-da-europa/a-15905514 <acesso, 2021>.

\section{REFERÊNCIAS}

APLICATIVO VERDES-PT. Registro ISNB 978-85-903805-3-5. APP Verdes Portugal: Universidade de Coimbra, 2018.

BASE DE DADOS DE PORTUGAL. Portada. Disponível em: www.pordata.pt. Acesso em: 11 fev. 2020

CRUZ, F. Nuno; CUNHA, M. Rui. Análise ecónomica do sistema da reciclagem em Portugal. Revista Sanit. Engenharia Ambiental, v. 19, n. 3, 2014.

DUSTON, Thomas, E. Recycling Solid Waste - The First Choice for Private and Public Sector Management. London: Quorum Books, 1993. 


\section{RECIMA21 - REVISTA CIENTÍFICA MULTIDISCIPLINAR}

VIABILIDADE ECONÔMICA DA RECICLAGEM DOS RESÍDUOS DOMÉSTICOS DA CIDADE DE COIMBRA - UTILIZANDO O APLICATIVO VERDES-PT Marcio Magera Conceição, Joelma Telesi Pacheco Conceição, Alessandro Rezende da Silva, Maria Fatima Alves, Marcelo Dourado Sales, Maurício Lamano Ferreira

INE. Instituto Nacional de Estatística. Disponível em: www.ine.pt. Acesso em: 11 fev. 2020.

LEFF, Enrique. Epistemologia ambiental. São Paulo: Cortez, 2001.

MAGERA, M. Os caminhos do lixo: da obsolescência programada à logística reversa. 2. ed. Campinas: Átomo Alinea, 2015.

MAGERA, M. Os empresários do lixo: um paradoxo da modernidade. 2. ed. Campinas: Átomo Alinea, 2005.

MAGERA, M. The value of the gargage - an analysis of the viability economic recycling of solid waste urbano of Portugal. Revista Educação Ensino, v. 13, n. 2, 2018. Disponível em: http://revistas.ung.br/index.php/educacao/article/view/3571

MAGERA, M. Viabilidade econômica da reciclagem dos resíduos urbanos da cidade de Lisboa -PT uma análise utilizando o aplicativo verdes-pt. Revista Research Society and Development, v. 9, n. 5, 2020. Disponível em: https://rsdjournal.org/index.php/rsd/article/view/2961/5363

TRADING-ECONOMICS. Portugal: balança comercial. https://pt.tradingeconomics.com/portugal/balance-of-trade. Acesso em: 2021. 


\section{RECIMA21 - REVISTA CIENTÍFICA MULTIDISCIPLINAR}

VIABILIDADE ECONÔMICA DA RECICLAGEM DOS RESÍDUOS DOMÉSTICOS DA CIDADE DE COIMBRA - UTILIZANDO O APLICATIVO VERDES-PT Marcio Magera Conceição, Joelma Telesi Pacheco Conceição, Alessandro Rezende da Silva, Maria Fatima Alves, Marcelo Dourado Sales, Maurício Lamano Ferreira

ANEXOS - Relatórios da cidade de Coimbra, enviado por e-mail, pelo Aplicativo VERDES-PT

VERDES- +55 11 99762-4781 | Página 1 de 1

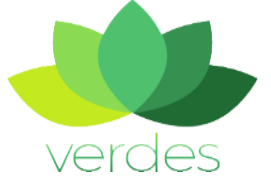

VERDES PT 2018

ISBN - 978-85-903805-3-5

Responsável: Prof. Dr. Márcio Magera Conceição PhD E-mail: magera1963@gmail.com

\section{Coimbra - Resultados Finais}

\begin{tabular}{|l|}
\hline \multicolumn{1}{|c|}{ Economia possivel (ou potencial) de ser conseguida anualmente coma reciclagem } \\
\hline Total obtido pela reciclagem: $€ 16.436 .805,49$ \\
\hline Total Perdido pela não reciclagem: $€ 14.580 .626,29$ \\
\hline Total: $€ 31.017 .431,77$ \\
\hline
\end{tabular}

\begin{tabular}{|l|}
\hline \multicolumn{1}{|c|}{ Empregos de 1 Salário Minimo } \\
\hline Conseguido como processo da reciclagem: 2.362 \\
\hline Perdidos pela não reciclagem dos resíduos urbanos: 2.095 \\
\hline
\end{tabular}

\begin{tabular}{|l|}
\hline \multicolumn{1}{|c|}{ Economia obtida anualmente pela reciclagem } \\
\hline Custo da reciclagem $€ 3.344 .240,01$ \\
\hline Custo evitado coma coleta: $€ 953.727,71$ \\
\hline Ganho de energia elétrica: $€ 3.714 .508,52$ \\
\hline Ganho de matéria-prima: $€ 14.484 .065,50$ \\
\hline Ganho de água: $€ 628.743,76$ \\
\hline Total: $€ 16.436 .805,49$ \\
\hline
\end{tabular}

\begin{tabular}{|l|}
\hline \multicolumn{1}{|c|}{ Economia perdida anualmente pela não-reciclagem } \\
\hline Custo da reciclagem $€ 2.215 .080,29$ \\
\hline Custo evitado coma coleta: $€ 631.708,08$ \\
\hline Ganho de energia elétrica: $€ 2.677 .781,63$ \\
\hline Ganho de matéria-prima: $€ 13.215 .141,66$ \\
\hline Ganho de água: $€ 271.075,20$ \\
\hline Total: $€ 14.580 .626,29$ \\
\hline
\end{tabular}




\section{RECIMA21 - REVISTA CIENTÍFICA MULTIDISCIPLINAR}

VIABILIDADE ECONÔMICA DA RECICLAGEM DOS RESÍDUOS DOMÉSTICOS DA CIDADE DE COIMBRA - UTILIZANDO O APLICATIVO VERDES-PT Marcio Magera Conceição, Joelma Telesi Pacheco Conceição, Alessandro Rezende da Silva, Maria Fatima Alves, Marcelo Dourado Sales, Maurício Lamano Ferreira

VERDES- +55 11 99762-4781 | Página 1 de 2
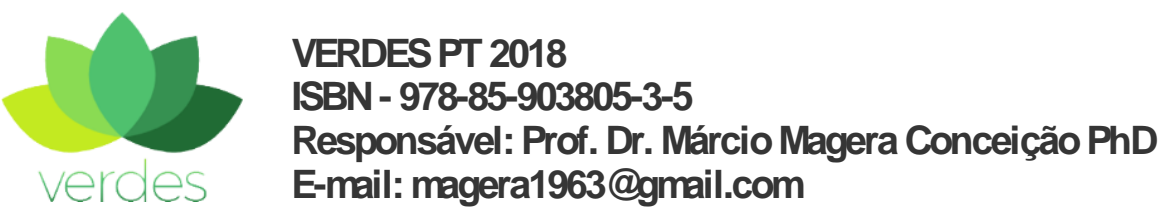

VERDES PT 2018

ISBN - 978-85-903805-3-5

Responsável: Prof. Dr. Márcio Magera Conceição PhD

E-mail:magera1963@gmail.com

\section{Coimbra - Impactos Macroambientais}

\begin{tabular}{|l|}
\multicolumn{1}{c|}{ Empregos de 1 Salário Mínimo (S.M) } \\
\hline Conseguido como processo da reciclagem: 2.362 \\
\hline Perdidos pela não reciclagem dos resíduos urbanos: 2.095 \\
\hline
\end{tabular}

\section{Quantidade de árvores que foram cortadas anualmente para gerar papel e cartão}

Árvores cortadas: 300.680

\section{Quantidade de árvores que foram poupadas anualmente como processo da reciclagem dos RSU}

Árvores poupadas: 210.476

Produção potencial anual de energia elétrica a partir do digestor do biogás, resíduos orgânicos

Produção de energia: $8.061,7 \mathrm{MWh}$

Gasto anual do município/país coma gestão dos resíduos sólidos urbanos

Gasto anual: $€ 6.536 .531,80$

Gasto anual de cada habitante coma gestão dos resíduos sólidos urbanos

Gasto anual por habitante: $€ 48,69$

\begin{tabular}{|l|}
\hline \multicolumn{1}{|c|}{ Volume recolhido por tipo de coleta } \\
\hline Coleta Seletiva: $9.804,8$ Ton \\
\hline Coleta indiferenciada: $55.560,5$ Ton \\
\hline
\end{tabular}

\section{Quantidade de casas que poderiam utilizar a energia elétrica gerada pelo biogás}

Casas beneficiadas: 7.329

\section{Lixômetro - Cuanto o municipio/país gerou de lixo até a data de hoje}

Lixo gerado: 7.444,3 Ton

Cuantidade da matéria prima bauxita usada para fazer o alumínio que foi descartado

Quantidade de bauxita: 470,6 Ton 


\section{RECIMA21 - REVISTA CIENTÍFICA MULTIDISCIPLINAR}

VIABILIDADE ECONÔMICA DA RECICLAGEM DOS RESÍDUOS DOMÉSTICOS DA CIDADE DE COIMBRA - UTILIZANDO O APLICATIVO VERDES-PT Marcio Magera Conceição, Joelma Telesi Pacheco Conceição, Alessandro Rezende da Silva, Maria Fatima Alves, Marcelo Dourado Sales, Maurício Lamano Ferreira

VERDES- +55 11 99762-4781 | Página 1 de 2

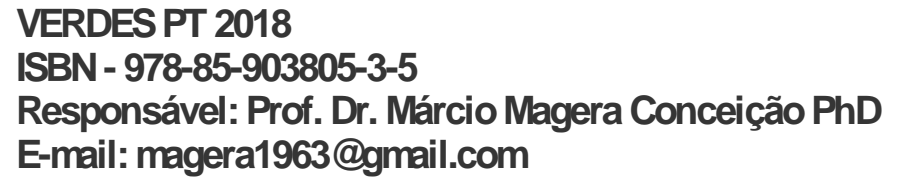

\begin{tabular}{|l|}
\hline Coimbra - Análise Macro-Ambiental \\
\hline Lixo Gerado por Habitante \\
\hline Por dia: $1,35 \mathrm{Kg}$ \\
\hline Por mês: $40,5 \mathrm{Kg}$ \\
\hline Por ano: $486,8 \mathrm{Kg}$ \\
\hline
\end{tabular}

\begin{tabular}{|l|}
\hline \multicolumn{1}{|c|}{ Geração total de lixo } \\
\hline Por dia: 181,57 Ton. \\
\hline Por mês: $5.447,1$ Ton. \\
\hline Por ano: $65.365,3$ Ton. \\
\hline
\end{tabular}

\begin{tabular}{|l|}
\hline \multicolumn{1}{|c|}{ Composição do Lixo em Toneladas } \\
\hline Orgânica: $24.185,1(37 \%)$ \\
\hline Papel e Cartão: $15.034,0(23 \%)$ \\
\hline Plástico: $8.497,4(13 \%)$ \\
\hline Vidro: $3.268,2(5 \%)$ \\
\hline Metais: $2.614,6(4 \%)$ \\
\hline Latas de Aço: $2.353,1(90 \%)$ \\
\hline Alumínio: $261,4(10 \%)$ \\
\hline
\end{tabular}

\section{Destino dos Resíduos Urbanos em toneladas/ano}

\begin{tabular}{l}
\hline Aterro: $18.955,9(29 \%)$ \\
\hline Tratamento MB: $18.302,2(28 \%)$ \\
\hline Valorização do Material: $7.190,1(11 \%)$ \\
\hline Valorização Energética: $14.380,3(22 \%)$ \\
\hline Tratamento Mecânico: $5.229,2(8 \%)$ \\
\hline Valorização Orgânica: $1.307,3(2 \%)$ \\
\hline
\end{tabular}

Índice de Reciclagem(2018)

\begin{tabular}{|l|}
\hline Papel e Cartão: $10.523,8(70 \%)$ \\
\hline Alumínio: $167,3(64 \%)$ \\
\hline Latas de Aço: $1.506,0(64 \%)$ \\
\hline Vidro: $1.928,2(59 \%)$ \\
\hline Plástico: $3.568,9(42 \%)$ \\
\hline Total gerado por mês: $17.694,3$ Ton. \\
\hline
\end{tabular}




\section{RECIMA21 - REVISTA CIENTÍFICA MULTIDISCIPLINAR}

VIABILIDADE ECONÔMICA DA RECICLAGEM DOS RESÍDUOS DOMÉSTICOS DA CIDADE DE COIMBRA - UTILIZANDO O APLICATIVO VERDES-PT Marcio Magera Conceição, Joelma Telesi Pacheco Conceição, Alessandro Rezende da Silva, Maria Fatima Alves, Marcelo Dourado Sales, Maurício Lamano Ferreira

VERDES- +55 11 99762-4781 | Página 1 de 3

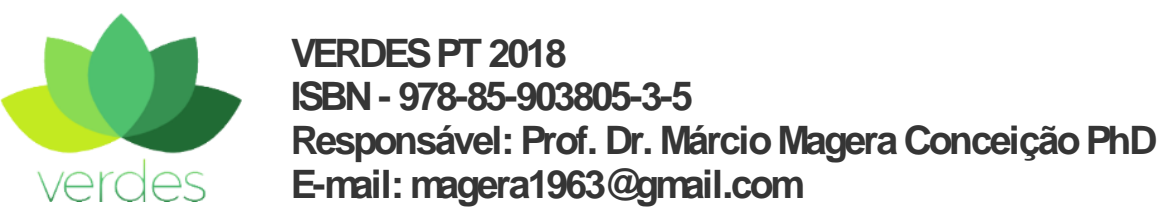

\section{Coimbra - Análise por Categoria}

\section{Alumínio}

\begin{tabular}{|l|}
\hline Consumo anual de latas: $16.760 .337,9$ \\
\hline Peso total em toneladas: 261,4 \\
\hline
\end{tabular}

\begin{tabular}{|l|}
\hline \multicolumn{1}{|c|}{ Economia coma reciclagem } \\
\hline Economia obtida anualmente pela reciclagem: $€ 195.698,53$ \\
\hline Economia perdida anualmente pela não-reciclagem: $€ 110.080,42$ \\
\hline Total por ano: $€ 305.778,96$ \\
\hline
\end{tabular}

\begin{tabular}{|l|}
\hline \multicolumn{1}{|c|}{ Total Reciclado (por ano) } \\
\hline Total Reciclado (por ano): 167,3 Ton. \\
\hline Total não reciclado: 94,1 Ton. \\
\hline
\end{tabular}

\begin{tabular}{|l|}
\hline \multicolumn{1}{|c|}{ Economia de Energia (por ano) } \\
\hline Economia obtida: $€ 155.538,08$ \\
\hline Economia perdida: $€ 87.490,17$ \\
\hline
\end{tabular}

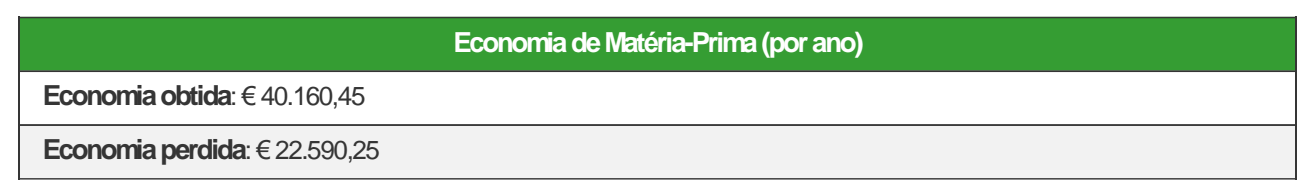

\section{Vidro}

\begin{tabular}{|l|}
\hline \multicolumn{1}{|c|}{ Economia coma reciclagem } \\
\hline Economia obtida anualmente pela reciclagem: $€ 607.792,87$ \\
\hline Economia perdida anualmente pela não-reciclagem: $€ 422.364,54$ \\
\hline Total por ano: $€ 1.030 .157,41$ \\
\hline
\end{tabular}

\begin{tabular}{|l|}
\hline \multicolumn{1}{|c|}{ Total Reciclado (por ano) } \\
\hline Total Reciclado (por ano): $1.928,2$ Ton. \\
\hline Total não reciclado: $1.339,9$ Ton. \\
\hline
\end{tabular}

